\title{
Septic Shock in Pregnancy Caused by Acute Chorioamnionitis: Diagnosis, Surgical Selection, and Hemodynamic Therapy
}

\section{Yi-si Zhao | Shi-hui Lin | Mu Zhang | Chuan-Jiang Wang | Fang Xu*}

*Correspondence: Fang Xu

Address: Department of Critical Care Medicine, The first affiliated hospital of Chongqing Medical University, Chongqing 400016, China

e-mail $\bowtie$ : xufang828@126.com

Received: 06 April 2020; Accepted: 15 April 2020

Copyright: (C) 2020 Zhao Yi-si. This is an open-access article distributed under the terms of the Creative Commons Attribution License, which permits unrestricted use, distribution, and reproduction in any medium, provided that the original work is properly cited.

\section{ABSTRACT}

Chorioamnionitis as an infectious disease that could cause sepsis in pregnancy which can be life threatening to both the mother and fetus. It needs early recognition and comprehensive management. In this paper, we report a case of septic shock caused by chorioamnionitis (39-year-old woman, 25 weeks pregnant), who received a successful multi-disciplinary treatment (MDT) from gynaecology and obstetrics, critical care medicine, and anesthesiology including antibiotics, fluid resuscitation, intensive care, and an emergency operation. From this case, we discuss clinical features, treatment timing, and methods of critical care for sepsis in pregnant woman.

Keywords: Sepsis, Septic shock, Chorioamnionitis, Pregnancy, Fluid resuscitation, Hysterectomy

\section{Introduction}

Sepsis in pregnancy is a disease which has a high mortality rate for both the mother and fetus. A global systematic analysis conducted by the World Health Organization (WHO) found that more than 261,000 (10.7\%) maternal deaths between 2003 and 2009 were caused by sepsis (Say et al., 2014). Although the etiology of maternal sepsis is unclear, it is known that some diseases during pregnancy, such as chorioamnionitis, can be fatal if the development of sepsis occurs, due to compromised immunity at that time (Turner, 2019). Chorioamnionitis is an infection with resultant inflammation of any combination of the amniotic fluid, placenta, fetus, fetal membranes, or decidua (Committee on Obstetric P, 2017). Chorioamnionitis must be taken seriously as it can result in pneumonia, sepsis, and fetal death. In this paper, we report a case of sepsis in pregnancy caused by chorioamnionitis which resulted in fetal death and maternal acute septic shock, and discusses some limitations of multi-disciplinary treatment (MDT), from the view point of critical care medicine. 


\section{Case Report}

A 39-year-old woman (gravida 7, para 1) presented at $252 / 7$ weeks of pregnancy with a 9day history of a few episodes of vaginal bleeding. Her temperature was $37.0^{\circ} \mathrm{C}$, pulse was 104 beats per minute, respiratory rate was 20 breaths per minute, and blood pressure (BP) was 103/73 mmHg. The fetal heart rate was 140 beats per minute. The ultrasonography diagnosed placenta previa. She was diagnosed with alpha-Thalassemia during this gestation. Obstetrical history included one cesarean section and five abortions. The patient did not smoke tobacco, drink alcohol, use illicit drugs, or feed pets.

The following morning her temperature was $38.4^{\circ} \mathrm{C}$ with pharyngalgia, no cough, and no abdominal pain. The patient's white blood cell (WBC) counts were 15430per mm3 (the laboratory examination results are shown in Table 1). The fetal heart rate was 180-210 beats per minute. She received an antiinfection therapy of $1.5 \mathrm{~g}$ of intravenous (IV) cefuroxime. During the second day of treatment, vaginal bleeding was continued and her temperature increased continuously. The index of infection including WBC, percentage of neutrophils (NEUT\%), C-reactive protein (CRP), and procalcitonin (PCT), remained high (Fig. 1a and Table 1). What followed was abdominal pain. The physical examination identified tenderness in the right lower abdominal quadrant and percussion pain in the kidney area. Ultrasound imaging showed a normal appendix and dilated bilateral ureters. Fetal heart rate remained at 180-210 beats per minute. These symptoms often suggest intrauterine infection, but the patient and her husband requested the pregnancy be continued despite advice from the Obstetrician to terminate the pregnancy.

On day five of hospitalization, after a modified anti-infective program (moxifloxacin, tinidazole), she was lassitude and silent. The patient exhibited respiratory distress with 33 breaths per minute. The monitor showed her pulse oxygen saturation (Sp02) was $85 \%$ on $3 \mathrm{~L} / \mathrm{min}$ nasal canula, BP was 75/40 $\mathrm{mmHg}$, and heart rate was 135 beats per minute. Her abdomen was tender and she was experiencing rebound pain. The fetal heart had disappeared suddenly, as confirmed by Doppler. She received urgent intensive therapy which included fluid infusion, high flow oxygen, and Norepinephrine (NE) to raise her BP. The patient was then transferred to the intensive care unit (ICU).

\section{Clinical course and management in ICU}

\section{Step1: Make the diagnosis and confirm the source of infection}

On the basis of the patient's symptoms, clinical variables and laboratory values (Table 1), and the critical care physician's evaluation of her vital signs and systemic conditions, a diagnosis of septic shock was made (SOFA=9, qSOFA=3). According to the sepsis in obstetrics score (S.O.S), this pregnant patient 
was scored 12 (temperature/+3, systolic blood pressure/+2, heart rate/+2, respiratory rate/+1, Sp02/+3, white blood cell count/+1, percentage of immature neutrophils not determined, Lac 0). A score of 12 indicated the patient was at a high risk of poor outcome (Albright et al., 2014).

Table 1: Clinical variables and laboratory values during the course of illness.

\begin{tabular}{|c|c|c|c|c|c|c|c|c|c|c|c|c|c|}
\hline \multirow[t]{2}{*}{ Variable } & \multicolumn{13}{|c|}{ Days of Illness } \\
\hline & 1 & 2 & 3 & 4 & 5 & 6 & 7 & 8 & 9 & 10 & 11 & 12 & 13 \\
\hline \multicolumn{14}{|l|}{ Clinical variables } \\
\hline Temperature $\left({ }^{\circ} \mathrm{C}\right)$ & 37.0 & 38.4 & 37.6 & 39.1 & 36.4 & 36.4 & 36.3 & 37.4 & 37.0 & 36.9 & 37.3 & 37.2 & 36.7 \\
\hline $\begin{array}{l}\text { Heart rate } \\
\text { (beats/min) }\end{array}$ & 104 & 115 & 112 & 89 & 130 & 114 & 67 & 107 & 103 & 98 & 103 & 97 & 98 \\
\hline $\begin{array}{l}\text { Respiratory rate } \\
\text { (breaths/min) }\end{array}$ & 20 & 20 & 20 & 20 & 25 & 17 & 19 & 22 & 19 & 15 & 16 & 23 & 19 \\
\hline $\begin{array}{l}\text { Blood pressure } \\
(\mathrm{mmHg})\end{array}$ & $103 / 73$ & $118 / 61$ & $97 / 66$ & $98 / 62$ & $73 / 53$ & $138 / 87$ & $130 / 79$ & $155 / 85$ & $143 / 56$ & $118 / 78$ & $138 / 79$ & $150 / 89$ & $121 / 69$ \\
\hline $\begin{array}{l}\text { Oxygen saturation } \\
(\%)\end{array}$ & - & - & - & - & 83 & 98 & 100 & 96 & 100 & 99 & 98 & 97 & - \\
\hline $\begin{array}{l}\text { Oxygen } \\
\text { concentration (\%) }\end{array}$ & 21 & 21 & 21 & 21 & 90 & 80 & 60 & 60 & 50 & 40 & 33 & 33 & 21 \\
\hline \multicolumn{14}{|l|}{ Laboratory values } \\
\hline $\begin{array}{l}\text { White cells } \\
\left(10^{\wedge} 9 / \mathrm{L}\right)\end{array}$ & 15.43 & 19.25 & 17.19 & 21.25 & 24.96 & 20.39 & 33.34 & 44.56 & 39.10 & 31.08 & 24.61 & 21.67 & 16.25 \\
\hline NEUT (\%) & 93.9 & 89.0 & 88.8 & 89.9 & 95.9 & 95.0 & 91.6 & 91.0 & 84.0 & 84.0 & 81.0 & 84.3 & 85.0 \\
\hline Hemoglobin (g/L) & 95 & 88 & 80 & 79 & 67 & 73 & 89 & 83 & 76 & 75 & 80 & 82 & 85 \\
\hline Hematocrit (\%) & 29.5 & 28.3 & 24.9 & 23.9 & 21.0 & 20.9 & 25.4 & 23.9 & 22.5 & 22.2 & 23.8 & 25.0 & 25.9 \\
\hline Platelets $\left(10^{\wedge} 9 / \mathrm{L}\right)$ & 247 & 242 & 192 & 182 & 149 & 77 & 68 & 67 & 70 & 154 & 238 & 335 & 401 \\
\hline AST (U/L) & 32 & - & - & - & 40 & 50 & 151 & 222 & 86 & 42 & 30 & 24 & 19 \\
\hline $\begin{array}{l}\text { Creatinine } \\
\text { (umol/L) }\end{array}$ & 42 & - & - & - & 144 & 184 & 124 & 241 & 345 & 301 & 156 & 93 & 79 \\
\hline $\mathrm{CRP}(\mathrm{mg} / \mathrm{L})$ & $>90.0$ & $>90.0$ & $>90.0$ & $>90.0$ & $>90.0$ & $>90.0$ & $>90.0$ & 56.1 & 58.4 & 58.0 & 66.9 & 62.9 & 54.5 \\
\hline Lactate $(\mathrm{mmol} / \mathrm{L})$ & - & - & - & - & 6.2 & 2.9 & 1.6 & 1.2 & 0.8 & 0.9 & 0.9 & 0.8 & - \\
\hline $\mathrm{pH}$ & - & - & - & - & 7.42 & 7.47 & 7.50 & 7.50 & 7.39 & 7.41 & 7.43 & 7.43 & - \\
\hline $\begin{array}{l}\text { Bicarbonate } \\
(\mathrm{mmol} / \mathrm{L})\end{array}$ & 21.3 & - & - & - & 16.2 & 26.2 & 25.0 & 27.3 & 28.5 & 29.2 & 30.5 & 28.5 & - \\
\hline Sodium (mmol/L) & 133 & - & - & - & 134 & 141 & 140 & 137 & 137 & 141 & 142 & 138 & - \\
\hline $\begin{array}{l}\text { Potassium } \\
(\mathrm{mmol} / \mathrm{L})\end{array}$ & 3.9 & - & - & - & 3.1 & 3.7 & 3.9 & 3.8 & 4.0 & 3.5 & 3.2 & 3.4 & - \\
\hline \multicolumn{14}{|l|}{ Scoring } \\
\hline APACHE II (score) & - & - & - & - & 16 & - & - & 19 & - & 10 & - & - & \\
\hline SOFA (score) & - & - & - & - & 9 & - & - & 7 & - & 8 & - & - & - \\
\hline
\end{tabular}


The computerized tomography (CT) showed there were no obvious signs of infection in the respiratory system or urinary system, which are the main causes of sepsis in pregnant women. Calculations from the CT scans, of the uterus and uterine contents on the pelvic plane, showed that there were no apparent inflammatory manifestations in the amniotic fluid, but a rough endometrium was observed, and the unclear delimitation between placenta and muscle layer was revealed (Fig. 1b). These observations provide some evidence for intra-amniotic infection (IAI). The CT image also showed the dead fetus' intracranial hemorrhage, which could be associated with mother's severe intrauterine infection (Aziz et al., 2009). In consideration of the CT images and her symptoms, including vaginal bleeding and abdominal pain, critical care doctors determined the source of infection was intrauterine and made the clinical diagnosis of chorioamnionitis, an inflammatory disease found in pregnant women. Three days later the pathological images showed that the smooth muscle of the uterus had edema, hemorrhage, and neutrophil infiltration. The patient's placental pathological images illustrated that leukocytes had extended through the full thickness of the chorioamniotic membrane, which is consistent with a diagnosis of chorioamnionitis (Fig. 1c).

\section{Step2: Create a surgical opportunity for the obstetrician}

For the treatment of sepsis, early surgical intervention allows for more rapid reduction of the associated symptoms (Kendle and Louis, 2018). The patient's situation was too poor to receive surgical intervention so anti-shock treatment was implemented to create the possibility for a surgical opportunity. The patient received broad-spectrum antibiotics (Imipenem and Cilastatin Sodium IV 2g + Linezolid IV 600mg, after her blood cultures samples was collected), ample fluid resuscitation, blood transfusion, application of vasopressors, hydrocortisone, and she was assisted with mechanical ventilation. A pulse index continuous cardiac output (PICCO, German, pc4000) and ultrasonic cardiac output monitor (USCOM, Australia, USCOM1A) were used to monitor her hemodynamic index, including cardiac output (CO), cardiac index (CI), global end-diastolic volume index (GEDI), extravascular lung water (EVLW), global ejection fraction (GEF), and systemic vascular resistance index (SVRI) (Table 2). Ten hours after $5500 \mathrm{ml}$ of liquid was infused into her circulatory system, the patient had a response to the fluid resuscitation (BP 98/61 mmHg, NE 1.6ug/(kg*min), Lac 4.5mmol/L, HR 126 beats per minute), but without urine. This gave her the opportunity to receive a subtotal hysterectomy in venous compliance with anesthesia.

Step3: Manage critically pregnant woman with excessive capacity recovery after operation

During the 2.5 hours of surgery, the patient hemorrhaged $2000 \mathrm{ml}$ and received $5050 \mathrm{ml}$ liquid (including crystalloid $1100 \mathrm{ml}$, colloid $1500 \mathrm{ml}$, cryoprecipitate $150 \mathrm{ml}$, fresh frozen plasma $900 \mathrm{ml}$, 
suspended red blood cells $1200 \mathrm{ml}$, and human albumin $200 \mathrm{ml}$ ). When the patient was sent back to ICU, she had stable vital signs (HR 118 beats per minute, BP 101/61 mmHg, NE $1.42 \mathrm{ug} /\left(\mathrm{kg}^{*} \mathrm{~min}\right)$ ). In ICU her total liquid input was $15500 \mathrm{ml}$ while her urinary volume was 55ml, the oxygenation index (OI) 214 $\mathrm{mmHg}$, Lac $10.7 \mathrm{mmol} / \mathrm{L}$, CI $1.5 \mathrm{~L} / \mathrm{min} / \mathrm{m}^{2}$, EVLW 9ml/kg, and SVRI 1130 dyn.s. $\mathrm{cm}^{2} . \mathrm{m}^{3}$, which suggested an overloaded capacity status and increased extravascular water in her lungs. Critical care physicians selected continuous renal replacement therapy (CRRT) to regulate fluid balance (the fluid management each day in ICU, showed in Fig. 1d).

\section{Prognosis}

The patient was weaned from mechanical ventilation and was extubated on day eight of her admission to ICU. The index of infection had begun to decline from day nine. She begun oral feeding on day 10. After two days (day 12), she was transferred from ICU to the obstetric ward and was discharged on day 22. Three months later, the patient returned to the obstetric clinic for follow-up and all laboratory values were recovering to normal ranges.
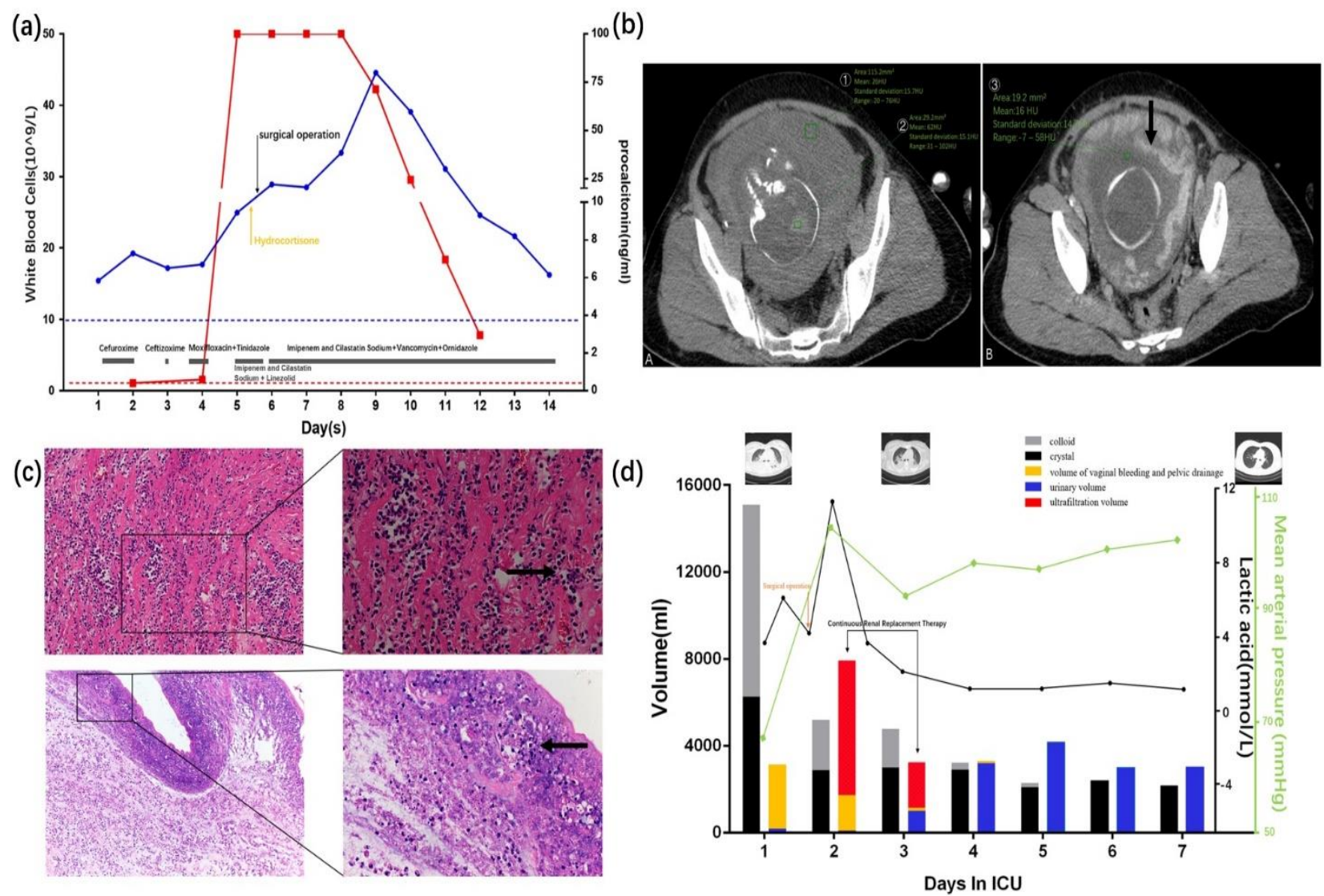

Figure 1(a): Timeline of index of infection, hydrocortisone therapy, surgical operation, and antimicrobial therapy in a patient with chorioamnionitis and sepsis of pregnancy. 
The change of procalcitonin (red line) and white blood cells (blue line) are shown in this diagram. The duration of antimicrobial therapy is shown by the gray bold lines. The gold arrow shows the time this patient received hormone therapy with hydrocortisone that appeared to raise the index of infection. The patient received surgical therapy (marked by a black arrow) at the fifth day of illness. On the same day, a new antimicrobial therapy regimen with Imipenem and Cilastatin Sodium, Vancomycin and Ornidazole, had been adopted (the longest bold line). The red dashed line represents the upper limit of the normal range for procalcitonin and the blue dashed line represents the upper limit of the normal range for white blood cells.

\section{(b). CT Images of the uterus}

The CT images (Pictures A and B) of the uterus were taken when the fetus was dead, after the patient was transferred in ICU. The data in (1)-(3) show the CT values of the muscle of the uterus, head of the fetus, and amniotic fluid. These three values show that this 25-weeks-pregnant uterus had rough endometrium, the delimitation between the placenta and muscle layer was not clear, the fetus had intracranial hemorrhage, and no apparent inflammation was detected in the amniotic fluid.

\section{(c). Pathobiology}

Two pathological images from the uterus of this patient (top images) show edema, hemorrhage and neutrophil infiltration in the smooth muscle of the uterus. The other two placental pathological images (bottom images) illustrate that leukocytes were infiltrating through the full thickness of the chorioamniotic membrane, which is consistent with a diagnosis of chorioamnionitis.

\section{(d). Fluid management}

This histogram shows the patient's fluid intake (left column) and output (right column) every day in ICU. The gray part of the right column shows the colloid which was injected into the patient each day, and the black part shows the crystal liquid. In the output column, the yellow section shows the bleeding volume from the vagina and volume of the pelvic drainage each day, the red section shows the ultrafiltration volume from the continuous renal replacement therapy (CRRT) and the blue section indicates the urinary volume. The tendency of lactate amounts (black broken line) and mean arterial pressure (MAP, showed by green line) reflect the degree of her shock and treatment effect. On the first day when the patient entered ICU, her fluid output volume only included vaginal bleeding and little urinary production, she had high lactate (Lac) and low MAP. This suggests shock with a deficiency of effective circulation. The patient received mass fluid resuscitation for treating her shock before surgery, 
as well as for preventing the shock aggravation during the intraoperative period. The orange arrow shows the time she received surgical therapy. On the first day after her operation, we began to give her the CRRT therapy (black line with double arrowheads) because of fluid overload. Three chest CT images were obtained separately before the surgical therapy, on the third day staying in ICU and two weeks after the operation, which showed the changes of this patient's pleural effusion. It suggests that fluid overload already existed in the early days, but had been absorbed with the treatment.

Table 2: Hemodynamic monitoring from the Pulse Index Continuous Cardiac Output (PICCO) and Ultrasonic Cardiac Output Monitor (USCOM).

\begin{tabular}{|c|c|c|c|c|}
\hline Variable & \multicolumn{4}{|c|}{ Days in ICU } \\
\hline $\operatorname{PICCO}(x \pm s)$ & 1 & 2 & 3 & 4 \\
\hline Cardiac index $\left(\mathrm{L} / \mathrm{min} / \mathrm{m}^{2}\right)$ & $3.56 \pm 0.16$ & $4.15 \pm 1.02$ & $3.15 \pm 0.04$ & $4.22 \pm 0.00$ \\
\hline Global end-diastolic volume index $\left(\mathrm{ml} / \mathrm{m}^{2}\right)$ & $722 \pm 18$ & $733 \pm 18$ & $601 \pm 162$ & $498 \pm 0$ \\
\hline Extravascular lung water (ml/kg) & $14 \pm 1$ & $9 \pm 1$ & $6 \pm 3$ & $5 \pm 0$ \\
\hline Global ejection fraction (\%) & $17 \pm 0$ & $20 \pm 4$ & $25 \pm 8$ & $29 \pm 0$ \\
\hline Systemic vascular resistance index $\left(\right.$ dyn.s.cm $\left.{ }^{2} . \mathrm{m}^{3}\right)$ & $748 \pm 324$ & $1486 \pm 148$ & $1897 \pm 467$ & $1522 \pm 0$ \\
\hline USCOM & & & & \\
\hline Velocity time integral $(\mathrm{cm})$ & - & 22 & 19 & 24 \\
\hline Percentage of ejection time (\%) & - & 61 & 57 & 56 \\
\hline Flow time corrected (ms) & - & 442 & 387 & 382 \\
\hline Stroke volume $\left(\mathrm{cm}^{3}\right)$ & - & 58 & 50 & 65 \\
\hline Stroke volume index $\left(\mathrm{ml} / \mathrm{m}^{2}\right)$ & - & 34 & 30 & 39 \\
\hline Cardiac output (L/min) & - & 6.7 & 6.4 & 8.3 \\
\hline Cardiac index $\left(\mathrm{L} / \mathrm{min} / \mathrm{m}^{2}\right)$ & - & 4.0 & 3.9 & 5.0 \\
\hline Systemic vascular resistance index (dyn.s. $\left.\mathrm{cm}^{2} \cdot \mathrm{m}^{3}\right)$ & - & 1883 & 1138 & 1276 \\
\hline Stroke volume variation (\%) & - & 31 & 33 & 35 \\
\hline Oxygen delivery (ml/min) & - & 640 & 682 & 876 \\
\hline
\end{tabular}

\section{Discussion}

We reported a case of septic shock caused by chorioamnionitis (39-year-old woman, 25 2/7 weeks pregnant), which resulted in an extremely adverse process with a successful outcome. From this case, we 
discussed the aim and the methods of the management of sepsis/septic shock in pregnancy, a population with special physiology and pathophysiology. Along with the deep understanding of sepsis, the latest definition (sepsis-3) describes the relationship between infection, host, and organ failure (Singer et al., 2016). Due to the refinement of pathophysiology in sepsis, patients with special physiological conditions, such as pregnancy, warrant additional focus (Bonet et al., 2017). Maternal sepsis, which is a lifethreatening condition defined as organ dysfunction resulting from infection during pregnancy, childbirth, post-abortion, or the postpartum period, (Queiros da Mota et al., 2013) was a new and important issue for obstetricians. Alongside SOFA and qSOFA, physicians could use S.O.S., a sepsis scoring system, which was designed specifically to reliably identify these patients at high risk for admission to the ICU (Albright et al., 2014). Furthermore, the specific immune response during pregnancy could increase the susceptibility and severity of infection (Pazos et al., 2012). A pregnant woman tends to show a general immune suppression for making the allogeneic fetus, to avoid the immune rejection.10-12 Additional research has also found that pregnancy results in an extensive T helper cell 2 (Th2)-biased immunity (Erlebacher, 2001; Szekeres-Bartho and Schindler, 2019). In this situation, the immunosuppression and the Th2 dominant immune response both cause a severe progression of sepsis (Hotchkiss et al., 2016; Xue et al., 2019). Moreover, maternal organs exhibit hyperemia, especially the uterus, which can promote the spread of infection.

Chorioamnionitis is a cause of sepsis which has a high maternal mortality rate, (Committee on Obstetric P, 2017) a high risk of neonatal sepsis, intracranial hemorrhage, respiratory distress syndrome, and fetal death.5 However, it is very difficult to make a clear diagnosis, especially in the early stage of sepsis, as the normal physiological parameters of pregnancy often overlap the systemic inflammatory response in chorioamnionitis (Bauer et al., 2014). Simultaneously, the search for pathogenic evidence of chorioamnionitis is very difficult (Catano Sabogal et al., 2018). In the case presented, the patient had a fever, without organ dysfunction, and no bacterial cultures were positive during hospitalization. This was potentially because the obstetrician made the clinical decision to administer the patient antibiotics for treating premature rupture of fetal membranes. The use of antibiotics prior to pathogen testing would reduce the positive identification of sepsis (Zadroga et al., 2013). Also, the culture for fastidious organisms, such as mycoplasmas, the most common organisms associated with chorioamnionitis, has low sensitivity (Queiros da Mota et al., 2013). In consideration of the impact on the fetus, CT scans of pregnant women are rare, unless it was absolutely necessary (and authorization was obtained from the pregnant woman or family members) (Dauer et al., 2012). It was undoubtedly necessary to find the source of infection through CT, the fastest and most intuitive imaging examination, after the vital signs of the fetus disappeared and the mother's life was threatened by septic shock. The pathological CT images of the 
uterus and placenta made the diagnosis clear (Fig. 1c). Clinical diagnosis (in early stage) combined with pathology (gold standard) is still the main method for diagnosing chorioamnionitis (Higgins et al., 2016).

Currently, the management of pregnancy-associated sepsis is based on the treatment strategies for sepsis in non-pregnant patients (Padilla and Palanisamy, 2017). Thus, the critical care physicians were required to join the treatment team with the obstetricians in the case presented. Firstly, the treatment focus was on controlling the infection and supporting the organs, instead of improving effectiveness of the body's reaction. Finding and treating the infectious source is the key to the treatment of sepsis (Marshall et al., 2004). In this case, performing a cesarean section or subtotal hysterectomy as rapidly as possible was significantly more important than antimicrobial treatment. Missing the surgical opportunity would have resulted in a poorer outcome by increasing the symptoms associated with the infection. Hence, most physicians believe that sooner is better, as long as the patient can tolerate the intervention (Plante, 2016). However, the transport of ill patients has many risks, including increasing the patient's heart rate, increased partial pressure of carbon dioxide, and reduced blood pressure. In addition to these potential changes, the risks of equipment-related accident, such as device detachment, dislodgement, or malfunction during transport, are also considerations, especially with invasive mechanical ventilation (Janz et al., 2017; Smith et al., 1990; Braman et al., 1987). Hypotension and hypoxia could restrict the opportunity of either surgery or moving to the operation room (Fromm and Dellinger, 1992). As a result, more obstetricians hesitate, debating whether an emergency operation is necessary. It has been proposed that critically ill patients receive emergency surgery at the ICU's bedside, in order to avoid the risk of transport. However, operation at the bedside remains less well studied and studies that have investigated it have found it has worse outcomes (Martin et al., 2018). Therefore, patients need an accurate evaluation by a MDT which includes physicians in gynaecology and obstetrics, critical care medicine and anesthesiology (Rosen et al., 2018).

In the case presented, termination of pregnancy and hysterectomy were required to remove the source of infection, and prevent progression of the infection. Adequate perioperative preparation, especially the management of fluid, was a key intervention for decreasing the failure rate of the operation. Positive fluid balance is an independent risk factor for mortality in a patient experiencing sepsis, especially during pregnancy when they are particularly susceptible to fluid overload. The increased blood volume in pregnancy with a single fetus is more than $48 \%$ of a non-pregnant woman and d red blood cells are increased more than 32\% (Kendle and Louis, 2018; de Oliveira et al., 2015; Brotfain et al., 2016; Pritchard, 1965). In this case, we used $1050 \mathrm{ml}$ IV fluid during resuscitation in the first 1 hour of admission to the ICU, and $2550 \mathrm{ml}$ fluid during the first 3 hours according to the "Surviving Sepsis Campaign" published in 2016 (Rhodes et al., 2017). However, based on the updated "Surviving Sepsis 
Campaign" published in 2018, this amount was not sufficient to reach the standard 1 hour bundle (Levy et al., 2018). The septic patient in mention was a pregnant woman, so it was questionable whether applying these principles of fluid resuscitation was appropriate in perinatology. It was unclear whether these volumes in a critically ill woman at 26 weeks of pregnancy would cause circulation volume overload. At that time, multiple reflections on tissue perfusion (MAP, Lac) and hemodynamics (EVLW, CI and so on) were at a terrible level. Some studies showed that poor scores on above index were independent predictors of mortality in septic shock patients (Houwink et al., 2016; Wang et al., 2016; Jozwiak et al., 2013). Therefore, the patient needed to reach the minimum condition for transfer or surgery, as soon as possible and large doses of liquid and vasoactive drugs in a short period became an inevitable choice (Kendle and Louis, 2018; Levitov et al., 2016). We also applied vasopressors to achieve a MAP above 65 mmHg. Critical care physicians monitored the patient's MAP, lactate (Okorie and Dellinger, 2011), performed invasive and non-invasive hemodynamic monitoring, and cardiac ultrasound (Supplementary Fig. 1) to monitor her body's reaction during fluid therapy. The anesthesiologist also used hemodynamic monitoring in surgery (Gelb et al., 2018). Postoperative fluid management is a continuous challenge, which was accompanied by special physiological changes after childbirth. The blood volume of the patient increased due to the absorption of interstitial fluid and reduction in the mechanical compression of the vena cava (Pritchard, 1965; Kerr, 1965; Tihtonen et al., 2005). CRRT can play a role in protecting the heart from overload with fluid and adjusting body fluid balance (Macedo and Mehta, 2016). Currently, visualization is an important trend in ICU monitoring. In the new clinical management guidelines for obstetrician-gynecologists by the American College of Obstetricians and Gynecologists (ACOG), the methods of visualization for critical ill non-pregnant such as ultrasonography was mentioned (ACOG Practice Bulletin No. 211, 2019). In the case presented, critical care doctors used cardiac ultrasonography to assess hemodynamic instability, heart function, blood volume, and degree of shock. Those measures can directly reflect the patient's response to fluid management (Levitov et al., 2016).

In summary, this case report shows that chorioamnionitis can occur without early specific symptoms and can be rapidly aggravated, causing sepsis. Sepsis of pregnancy also has high risk of mortality and numerous complications if early recognition and diagnosis does not occur. However, it can be treated successfully with comprehensive management including strict monitoring of the patient and combined treatment, taking into account the recommendations for non-pregnant sepsis patients and the physiological changes that occur during pregnancy. Above all, rapidly controlling the source of infection is still the therapeutic target, which should not be missed. However, care should be taken to correctly identify the source of infection. This means an obstetrician should pay close attention to critical care in pregnancy. 


\section{Acknowledgements}

This study was supported by a Special fund of social undertakings and people's livelihood guarantee of Chongqing science and technology commission (cstc2017shmsA130072, to FX) and a Medical research project of Chongqing City Health and Family Planning committee fund (2017ZDXM007 to FX). We thank XM Z, ZB X, XM H and H Z for offering suggestions on the manuscript. We also wish to thank JL L for the operation in USCOM and ultrasound, and to LY D for the assistance in pathology.

Statement of Ethics: This patient had given their written informed consent to publish their case.

Disclosure Statement: The authors had no conflicts of interest to declare.

(a)

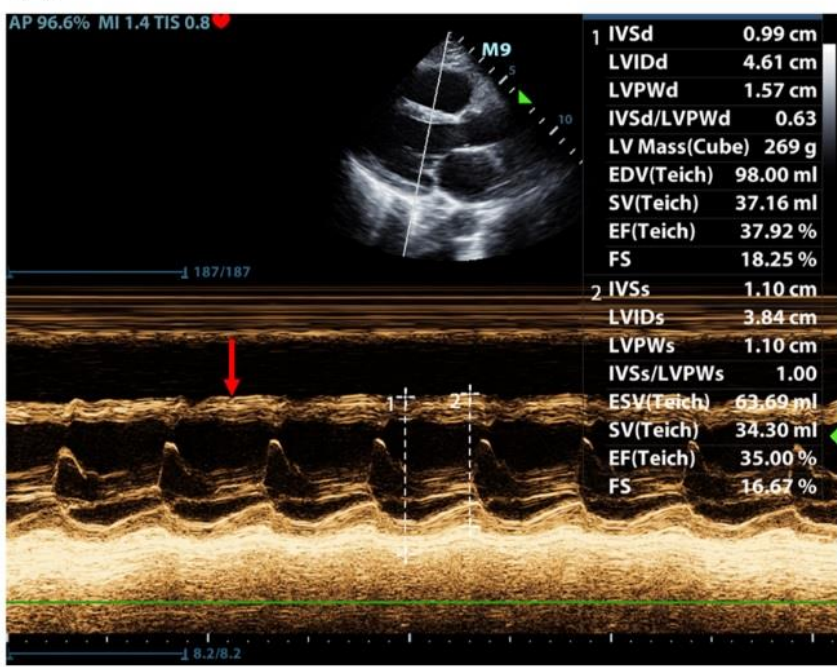

(b)

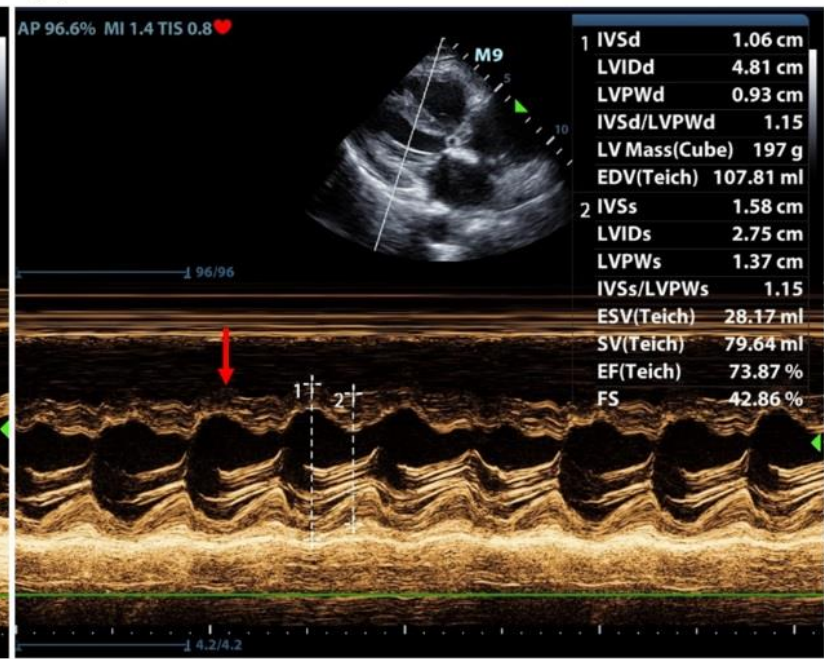

Supplementary Figure 1: Cardiac Ultrasound: Supplementary figure 1 includes two images showing the cardiac ultrasound from this patient at different times. Image (a) was taken on the first day after the operation and image (b) was taken on the third day after the operation. The image (a) shows left ventricular ejection fraction (EF) of 35\%, fractional shortening (FS) of approximately $16.67 \%$, stroke volume (SV) $34.40 \mathrm{ml}$, left ventricular end systolic volume (ESV) approximately $63.69 \mathrm{ml}$ and the pulsatile range (red arrow) decreasing when compared to image (b). In image (b), the EF reached 73.87\%, FS 42.86\%, SV $79.64 \mathrm{ml}$, and ESV $28.17 \mathrm{ml}$, which demonstrates that her left ventricular function was improved between post-operative day one and day three. 


\section{References}

ACOG Practice Bulletin No. 211: Critical Care in Pregnancy. Obstet Gynecol 2019; 133: e303-e319.

Albright CM, Ali TN, Lopes V, Rouse DJ, Anderson BL. The Sepsis in Obstetrics Score: a model to identify risk of morbidity from sepsis in pregnancy. Am J Obstet Gynecol 2014; 211: e31-38.

Aziz N, Cheng YW, Caughey AB. Neonatal outcomes in the setting of preterm premature rupture of membranes complicated by chorioamnionitis. J Matern Fetal Neonatal Med 2009; 22: 780-784.

Bauer ME, Bauer ST, Rajala B, MacEachern MP, Polley LS, Childers D, Aronoff DM. Maternal physiologic parameters in relationship to systemic inflammatory response syndrome criteria: a systematic review and meta-analysis. Obstet Gynecol 2014; 124: 535-541.

Bonet M, Nogueira Pileggi V, Rijken MJ, Coomarasamy A, Lissauer D, Souza JP, Gulmezoglu AM. Towards a consensus definition of maternal sepsis: results of a systematic review and expert consultation. Reprod Health 2017; $14: 67$.

Braman SS, Dunn SM, Amico CA, Millman RP. Complications of intrahospital transport in critically ill patients. Ann Intern Med 1987; 107: 469-473.

Brotfain E, Koyfman L, Toledano R, Borer A, Fucs L, Galante O, Frenkel A, Kutz R, Klein M. Positive fluid balance as a major predictor of clinical outcome of patients with sepsis/septic shock after ICU discharge. Am J Emerg Med 2016; 34: 2122-2126.

Catano Sabogal CP, Fonseca J, Garcia-Perdomo HA. Validation of diagnostic tests for histologic chorioamnionitis: Systematic review and meta-analysis. Eur J Obstet Gynecol Reprod Biol 2018; 228: 13-26.

Committee on Obstetric P. Committee Opinion No. 712: Intrapartum Management of Intraamniotic Infection. Obstetrics and gynecology 2017; 130: e95-e101.

Dauer LT, Thornton RH, Miller DL, Damilakis J, Dixon RG, Marx MV, Schueler BA, Vañó E, Venkatesan AM, Bartal G, Tsetis D. Radiation management for interventions using fluoroscopic or computed tomographic guidance during pregnancy: a joint guideline of the Society of Interventional Radiology and the Cardiovascular and Interventional Radiological Society of Europe with Endorsement by the Canadian Interventional Radiology Association. J Vasc Interv Radiol 2012; 23: 19-32.

de Oliveira FS, Freitas FG, Ferreira EM, de Castro I, Bafi AT, de Azevedo LC, Machado FR. Positive fluid balance as a prognostic factor for mortality and acute kidney injury in severe sepsis and septic shock. J Crit Care 2015; 30: 97-101.

Erlebacher A. Why isn't the fetus rejected? Current opinion in immunology 2001; 13: 590-593.

Fromm RE. Jr., and Dellinger RP. Transport of critically ill patients. J Intensive Care Med 1992; 7: 223-233.

Gelb AW, Morriss WW, Johnson W, Merry AF, International Standards for a Safe Practice of Anesthesia W. World Health Organization-World Federation of Societies of Anaesthesiologists (WHO-WFSA) International Standards for a Safe Practice of Anesthesia. Can J Anaesth 2018; 65: 698-708.

Higgins RD, Saade G, Polin RA, Grobman WA, Buhimschi IA, Watterberg K, Silver RM, Raju TN. Evaluation and Management of Women and Newborns With a Maternal Diagnosis of Chorioamnionitis: Summary of a Workshop. Obstet Gynecol 2016; 127: 426-436.

Hotchkiss RS, Moldawer LL, Opal SM, Reinhart K, Turnbull IR, Vincent JL. Sepsis and septic shock. Nat Rev Dis Primers 2016; 2: 16045 . 
Houwink AP, Rijkenberg S, Bosman RJ, van der Voort PH. The association between lactate, mean arterial pressure, central venous oxygen saturation and peripheral temperature and mortality in severe sepsis: a retrospective cohort analysis. Critical care 2016; 20: 56.

Janz DR, Khan YA, Mooney JL, Semler MW, Rice TW, Johnson JL, deBoisblanc BP. Effect of Interhospital ICU Relocation on Patient Physiology and Clinical Outcomes. J Intensive Care Med 2017: 885066617726754.

Jozwiak M, Silva S, Persichini R, Anguel N, Osman D, Richard C, Teboul JL, Monnet X. Extravascular lung water is an independent prognostic factor in patients with acute respiratory distress syndrome. Crit Care Med 2013; 41: 472-480.

Kendle AM and Louis J. Recognition and Treatment of Sepsis in Pregnancy. J Midwifery Womens Health 2018; 63: $347-351$.

Kerr MG. The Mechanical Effects of the Gravid Uterus in Late Pregnancy. J Obstet Gynaecol Br Commonw 1965; 72: 513529.

Levitov A, Frankel HL, Blaivas M, Kirkpatrick AW, Su E, Evans D, Summerfield DT, Slonim A, Breitkreutz R, Price S, McLaughlin M. Guidelines for the Appropriate Use of Bedside General and Cardiac Ultrasonography in the Evaluation of Critically Ill Patients-Part II: Cardiac Ultrasonography. Crit Care Med 2016; 44: 1206-1227.

Levy MM, Evans LE, Rhodes A. The Surviving Sepsis Campaign Bundle: 2018 update. J Intensive Care Med 2018; 44: 925928.

Macedo E and Mehta RL. Continuous Dialysis Therapies: Core Curriculum 2016. Am J Kidney Dis 2016; 68: 645-657.

Marshall JC, Maier RV, Jimenez M, Dellinger EP. Source control in the management of severe sepsis and septic shock: an evidence-based review. Crit Care Med 2004; 32: S513-526.

Martin ND, Patel SP, Chreiman K, Pascual JL, Braslow B, Reilly PM, Kaplan LJ. Emergency Laparotomy in the Critically Ill: Futility at the Bedside. Crit Care Res Pract 2018; 2018: 6398917.

Okorie ON and Dellinger P. Lactate: biomarker and potential therapeutic target. Crit Care Clin 2011; 27: 299-326.

Padilla C and Palanisamy A. Managing Maternal Sepsis: Early Warning Criteria to ECMO. Clin Obstet Gynecol 2017; 60: 418-424.

Pazos M, Sperling RS, Moran TM, Kraus TA. The influence of pregnancy on systemic immunity. Immunologic research 2012; 54: 254-261.

Plante LA. Management of Sepsis and Septic Shock for the Obstetrician-Gynecologist. Obstet Gynecol Clin North Am 2016; 43: 659-678.

Pritchard JA. Changes in the Blood Volume during Pregnancy and Delivery. Anesthesiology 1965; 26: 393-399.

Queiros da Mota V, Prodhom G, Yan P, Hohlfheld P, Greub G, Rouleau C. Correlation between placental bacterial culture results and histological chorioamnionitis: a prospective study on 376 placentas. J Clin Pathol 2013; 66: 243-248.

Rhodes A, Evans LE, Alhazzani W, Levy MM, Antonelli M, Ferrer R. Surviving Sepsis Campaign: International Guidelines for Management of Sepsis and Septic Shock: 2016. J Intensive Care Med 2017; 43: 304-377.

Rosen MA, DiazGranados D, Dietz AS, Benishek LE, Thompson D, Pronovost PJ, Weaver SJ. Teamwork in healthcare: Key discoveries enabling safer, high-quality care. Am Psychol 2018; 73: 433-450. 
Say L, Chou D, Gemmill A, Tuncalp O, Moller AB, Daniels J, Gulmezoglu AM, Temmerman M, Alkema L. Global causes of maternal death: a WHO systematic analysis. The Lancet. Global health 2014; 2: e323-333.

Singer M, Deutschman CS, Seymour CW, Shankar-Hari M, Annane D, Bauer M, Bellomo R, Bernard GR, Chiche JD, Coopersmith CM, Hotchkiss RS. The Third International Consensus Definitions for Sepsis and Septic Shock (Sepsis-3). Jama 2016; 315: 801-810.

Smith I, Fleming S, Cernaianu A. Mishaps during transport from the intensive care unit. Crit Care Med 1990; $18: 278-281$.

Szekeres-Bartho J and Schindler AE. Progestogens and immunology. Best Pract Res Clin Obstet Gynaecol 2019; 60: 17-23.

Tihtonen K, Koobi T, Yli-Hankala A, Uotila J. Maternal hemodynamics during cesarean delivery assessed by whole-body impedance cardiography. Acta obstetricia et gynecologica Scandinavica 2005; 84: 355-361.

Turner MJ. Maternal sepsis is an evolving challenge. Int J Gynaecol Obstet 2019; 146: 39-42.

Wang H, Cui N, Su L, Long Y, Wang X, Zhou X, Chai W, Liu D. Prognostic value of extravascular lung water and its potential role in guiding fluid therapy in septic shock after initial resuscitation. J Crit Care 2016; 33: 106-113.

Xue M, Xie J, Liu L, Huang Y, Guo F, Xu J, Yang Y, Qiu H. Early and dynamic alterations of Th2/Th1 in previously immunocompetent patients with community-acquired severe sepsis: a prospective observational study. J Transl Med 2019; 17: 57.

Zadroga R, Williams DN, Gottschall R, Hanson K, Nordberg V, Deike M, Kuskowski M, Carlson L, Nicolau DP, Sutherland C, Hansen GT. Comparison of 2 blood culture media shows significant differences in bacterial recovery for patients on antimicrobial therapy. Clin Infect Dis 2013; 56: 790-797. 